\title{
ALMOST PERIODIC AND PERIODIC SOLUTIONS OF DIFFERENCE EQUATIONS
}

\author{
BY GEORGE R. SELL ${ }^{1}$
}

Communicated by H. A. Antosiewicz, November 12, 1965

1. Introduction. Let $W$ be an open set in $R^{r}$, real $r$-space, and let $f: W \times I \rightarrow R^{r}$ be a continuous function, where $I$ denotes the integers. Consider the difference equation

$$
y_{n+1}=f\left(y_{n}, n\right) .
$$

It is easy to see that for every point $(y, N)$ in $W \times I$ there is a solution $\phi(n)$ of (1) that satisfies $\phi(N)=y$. This solution is defined and unique on some set $N \leqq n<N_{\infty}$, where $N_{\infty}$ is maximal. (That is, either $N_{\infty}=\infty$ or $\phi\left(N_{\infty}-1\right) \notin W$.) The solution may or may not be continuable for $n<N$ but, even if it is, the continuation may not be unique. Let $\phi(y, f, n), 0 \leqq n<N_{\infty}(y, f)$, be the solution of (1) that satisfies $\phi(y, f, 0)$ $=y$.

One can view the function $f$ defined on $W \times I$ as the restriction of a continuous function defined on $W \times R$, where $R$ denotes the real numbers. This viewpoint will be assumed in the sequel.

Our problem is to find sufficient conditions such that Equation (1) has a periodic or almost periodic solution. We shall consider only functions $f(y, t)$ that are uniformly almost periodic. This means that $f$ is uniformly continuous on each set $K \times R$, where $K$ is compact in $W$, and for each $x, f(x, t)$ is Bohr almost periodic in $t$. Our principal result states that under certain stability conditions Equation (1) has an almost periodic solution. If $f$ is periodic in $t$, with integral period, then we are able to prove the existence of a periodic solution.

Let $f$ be a uniformly almost periodic function defined on $W \times I$ and let $\left\{f_{k}: k \in I\right\}$ be the space of translates of $f$, where $f_{k}(y, n)=f(y, k+n)$. The hull of $f, H(f)$, is defined to be the closure of $\left\{f_{k}: k \in I\right\}$ in the topology of uniform convergence on sets of the form $K \times I$, where $K$ is compact in $W$. (For the uniformly almost periodic functions this is the same as the closure of $\left\{f_{k}: k \in I\right\}$ in the compact-open topology [8].) In addition to Equation (1) we shall be interested in the solutions of

$$
y_{n+1}=f^{*}\left(y_{n}, n\right),
$$

where $f^{*} \in H(f)$.

\footnotetext{
1 This research was supported in part by NSF Grant No. GP-3904.
} 
2. Definitions and statement of results. A solution $\phi(n)$ of (1) is said to be positively compact if the set $\left\{\phi(n): 0 \leqq n<N_{\infty}\right\}$ lies in a compact set in $W$. (In this case $N_{\infty}=\infty$.) A solution $\phi(n), n \geqq 0$, is periodic if there is a $K \geqq 1$ such that $\phi(n+K)=\phi(n)$, for all $n \geqq 0$. A solution $\phi(n), n \geqq 0$, is almost periodic if for every $\epsilon>0$ the set

$$
\left\{k \in I^{+}:|\phi(n+k)-\phi(n)| \leqq \epsilon \text { for all } n \text { in } I^{+}\right\}
$$

is relatively dense in $I^{+}$, where $I^{+}=\{n: n \geqq 0\}$.

We now define three types of stability. First assume that $0 \in W$ and $f(0, n)=0, n \geqq 0$. Then $\phi(0, f, n)=0$ is the null solution of (1). We say that the null solution is:

(i) uniformly stable if there is a continuous, strictly increasing function $\alpha(r)$ defined for $0 \leqq r \leqq a$ with $\alpha(0)=0$ and such that

$$
\left|\phi\left(y, f_{k}, n\right)\right| \leqq \alpha(|y|), \quad(|y| \leqq a, 0 \leqq k, 0 \leqq n) ;
$$

(ii) uniformly asymptotically stable if there is an $\alpha(r)$ as in (i) and a positive, decreasing function $\sigma(n)$, with $\sigma(n) \rightarrow 0$ as $n \rightarrow \infty$, such that

$$
\left|\phi\left(y, f_{k}, n\right)\right| \leqq \alpha(|y|) \sigma(n), \quad(|y| \leqq a, 0 \leqq k, 0 \leqq n) ;
$$

(iii) stable under persistent disturbances if for every $\epsilon>0$ there is a $\delta>0$ such that

$$
\left|\phi\left(y, f_{k}+g_{k}, n\right)\right| \leqq \epsilon \quad(0 \leqq k, 0 \leqq n),
$$

whenever $|y| \leqq \delta$ and $g: W \times I \rightarrow R^{r}$ is continuous and satisfies

$$
|g(y, n)| \leqq \delta, \quad(|y| \leqq \epsilon, 0 \leqq n) .
$$

If $\phi(n)=\phi(y, f, n)$ is an arbitrary solution of (1), then we say that $\phi$ is-stable whenever the null solution of

$$
y_{n+1}=f\left(y_{n}+\phi(n), n\right)-f(\phi(n), n)
$$

is-stable.

We are now able to prove the following results:

TheOREM 1. Let $f: W \times R \rightarrow R^{r}$ be uniformly almost periodic. Assume that there exists a positively compact solution of (1) that is stable under persistent disturbances. Then there exists an almost periodic solution for every Equation (2) in $H(f)$. In particular, Equation (1) has an almost periodic solution.

Theorem 2. Let $f: W \times R \rightarrow R^{r}$ be continuous and periodic in $t$ with integral period.

(A) If there exists a positively compact solution of (1) that is uniformly stable, then there exists an almost periodic solution of (1). 
(B) If there exists a positively compact solution of (1) that is uniformly asymptotically stable, then Equation (1) has a periodic solution.

3 . Outline of proofs. These theorems are closely related to corresponding results $[1],[6],[7],[8],[9]$ for ordinary differential equations. The proofs consist of appropriate modifications of the pertinent results from these papers.

The arguments in the above cited papers are based on two facts, [1, Theorem 5] and [7, Theorems 1 and 2], from the topological dynamics of continuous flows. Here we need the analogous statements for discrete flows. These analogues are proved in the same manner. (See [2] for a unified treatment of this basic theory.)

In order to apply these results to the problem at hand, we (formally) define the mapping

$$
\pi\left(y, f^{*}, n\right)=\left(\phi\left(y, f^{*}, n\right), f_{n}^{*}\right), \quad(0 \leqq n) .
$$

The domain of $\pi$ is a subset of $W \times H(f) \times I^{+}$with range in $W \times H(f)$. When $W$ has the usual topology and $H(f)$ the compact-open topology, the function $\pi$ is a "local dynamical system," in the sense defined in [8] but appropriately modified for discrete flows. Let us note that if there is a solution $\phi(y, f, n)$ that is positively compact, then there is a nonempty subset $L B$ in $W \times H(f)$ with the property that $\pi$ is a (discrete) flow on $L B \times I^{+}$. (See [6] or [8].)

The remainder of the proof is essentially analogous to the corresponding arguments for ordinary differential equations. (See in particular, [1, Theorem 6], [6, Theorem 2] and [7, Theorem 4]. We omit the details.

One may ask whether the assumption of "stability under persistent disturbances" can be replaced by "uniform asymptotic stability." It is known [3] that for ordinary differential equations, uniform asymptotic stability is stronger than stability under persistent disturbances. This is proved by constructing a suitable Lyapunov function. These techniques have been extended to studying the null solution of difference equations [5], provided the function $f$ satisfies:

(i) $|f(y, n)-f(z, n)| \leqq K_{a}|y-z|,(0 \leqq n,|y| \leqq a,|z| \leqq a)$, where $K_{a} \geqq 0$, and

(ii) $|f(y, n)| \geqq \beta(|y|),(0 \leqq n,|y| \leqq a)$, where $\beta(r)$ is positive for $r>0$.

Unfortunately condition (ii) is somewhat unnatural, and it is difficult to test when one is examining an arbitrary solution. However, by demanding that the Lipschitz constant $K_{a}$ is small, we can eliminate condition (ii). 
LEMмA. Let $f(0, n)=0, n \geqq 0$, and assume that there is a $K, 0 \leqq K<1$, such that
$|f(y, n)-f(z, n)| \leqq K|y-z|$,
$(0 \leqq n,|y| \leqq a,|z| \leqq a)$.

If the null solution of (1) is uniformly asymptotically stable, then it is stable under persistent disturbances.

The proof consists of showing that if

$$
\xi(y, f, g, n)=\phi(y, f+g, n)-\phi(y, f, n),
$$

and $|y|$ is sufficiently small, then

$$
|\phi(y, f+g, n)| \leqq|\phi(y, f, n)|+\delta\left(1+K+\cdots+K^{n}\right), \quad n \geqq 0,
$$

where $g(y, n)$ satisfies (5). It follows then that if $|y|$ is sufficiently small, then

$$
|\phi(y, f+g, n)| \leqq \alpha(|y|) \sigma(n)+\delta(1-K)^{-1},
$$

which implies the desired conclusion.

Corollary. Assume that $f: W \times R \rightarrow R^{r}$ is uniformly almost periodic and satisfies (7) everywhere where $0 \leqq K<1$. If there is a positively compact solution of (1) that is uniformly asymptotically stable, then Equation (1), and every Equation (2) in $H(f)$, has an almost periodic solution.

4. Remarks. 1. These results are more general than the corresponding results of A. Halanay [4] who considered small perturbations of stable linear difference equations.

2. One could formulate a stronger form of Theorems 1 and 2 by requiring that the given solution be stable with respect to an appropriate set. However, it appears that the given hypotheses will be more practical for applications.

Added in proof. By constructing a suitable Lyapunov function, R. D. Driver [10] is able to prove the above lemma without assuming that $K<1$. It then follows that one can remove the restriction $K<1$ in the above corollary.

\section{REFERENCES}

1. L. G. Deysach and G. R. Sell, On the existence of almost periodic motions, Michigan Math. J. 12 (1965), 87-95.

2. W. Gottschalk and G. Hedlund, Topological dynamics, Amer. Math. Soc. Colloq. Publ. Vol. 36, Amer. Math. Soc., Providence, R. I., 1955.

3. W. Hahn, Theory and application of Lyapunov's direct method, Prentice-Hall, Englewood Cliffs, N. J., 1963. 
4. A. Halanay, Solutions périodiques et presque-périodiques des systèmes d'équations aux différence finies, Arch. Rational Mech. Anal. 12 (1963), 134-149.

5. - Quelques questions de la théorie de la stabilité pour les systèmes aux différences finies, Arch. Rational Mech. Anal. 12 (1963), 150-154.

6. R. K. Miller, Almost periodic differential equations as dynamical systems with applications to the existence of a.p. solutions, J. Differential Equations 1 (1965), 337345.

7. G. R. Sell, Periodic solutions and asymptotic stability. J. Differential Equations (to appear).

8. - Nonautonomous differential equations and topological dynamics. I. The basic theory, (to appear).

9. - Nonautonomous differential equations and topological dynamics. II. Limiting equations, (to appear).

10. R. D. Driver, Note on a paper of Halanay on stability for finite difference equations, Arch. Rational Mech. Anal. 18 (1965), 241-243.

UNIVERSITY OF MinNESOTA 